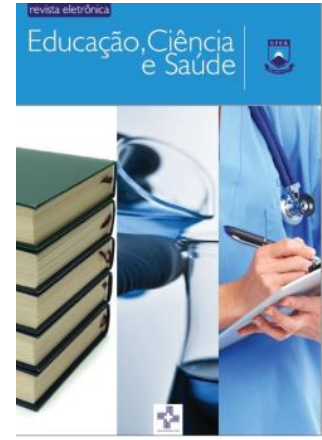

EDUCAÇÃO CIÊNCIA E SAÚDE

http://dx.doi.org/10.20438/ ecs.v8i1.382

\title{
CONDUTAS E INTERVENÇÕES NUTRICIONAIS FRENTE A PACIENTES OBESOS E INFECTADOS COM COVID-19
}

\author{
Emanuele Ferreira da Cruz ${ }^{1}$, Nayane Gabriela Ferreira Macedo e Silva ${ }^{1}$, \\ Nilcimelly Rodrigues Donato ${ }^{1}$, Vanessa Bordin Viera ${ }^{1}$, Edson Douglas Silva \\ Pontes ${ }^{1}$ \\ 1 Universidade Federal de Campina Grande, Brasil. \\ Email para correspondência: edsondspontes@gmail.com
}

\begin{abstract}
Resumo
A obesidade é uma doença crônico-degenerativa e inflamatória de origem multifatorial. A COVID-19 é uma doença causada por um vírus denominado SARS-CoV-2, que está sendo rapidamente disseminada entre a população global. A COVID-19 apresenta uma variação em seu aspecto clínico, podendo ser desde infecções assintomáticas a quadros grave. Objetivou-se realizar um levantamento bibliográfico sobre a possível correlação entre a obesidade e o COVID-19. Para tal, foi realizado uma revisão da literatura do tipo narrativa, em que utilizou-se artigos, guias e documentos oficiais de organizações da área de saúde e nutrição em língua inglesa e portuguesa publicados no período entre dezembro de 2019 a março de 2021 recuperados a partir da busca nos bancos de dados: PubMed, ScienceDirect e SciELO. Portanto, estudos realizados demonstram possuir uma relação entre a obesidade e os quadros mais graves da doença. Além disso, destaca-se o papel que a nutrição exerce nesse meio considerando as fases de prevenção, infecção e tratamento e as possíveis ferramentas nutricionais para tentar minimizar os impactos causados a saúde dos infectados e promover uma boa saúde e recuperação.
\end{abstract}

Palavras-chave: ferramentas nutricionais, sars-cov-2, obesidade.

\begin{abstract}
/ resumen / résumé
Obesity is a chronic-degenerative inflammatory disease of multifactorial origin. COVID-19 is a disease caused by a virus called SARS-CoV-2, which is spreading rapidly among the world's population. COVID-19 presents a variation in its clinical appearance, which can range from asymptomatic infections to severe conditions. The objective was to carry out a bibliographic survey on the possible correlation between obesity and COVID-19. For this, a narrative review of the literature was carried out, using articles, guides and official documents of health and nutrition organizations in English and Portuguese published in the period between December 2019 and March 2021, recovered from the search in the databases of data: PubMed, ScienceDirect. and SciELO. Studies show that there is a relationship between obesity and the most severe cases of the disease. In addition, the role that nutrition plays in this environment is highlighted, considering the stages of prevention, infection and treatment and possible nutritional tools to try to minimize the impacts caused on the health of those infected and promote good health and recovery.
\end{abstract}

Keywords: nutritional tools, sars-coV-2, obesity. 


\section{Introdução}

Segundo a Organização Mundial de Saúde (OMS, 2000), a obesidade é caracterizada pelo acúmulo anormal ou excessivo de gordura, que acarreta prejuízos à saúde dos indivíduos. Visto que, consequente ao sedentárismo se destaca com um papel central nas doenças crônicas não transmissíveis, pois propicia o desenvolvimento de outras comorbidades como diabetes tipo 2, dislipidemia, hipertensão, inflamação crônica e diferentes tipos de câncer (CHIAPPETTA et al., 2020; SALTIEL et al., 2017; MUSCOGIURI et al., 2020).

A doença causada pelo novo coronavírus (COVID-19) surgiu em Wuhan na China em dezembro de 2019 e se tornou rapidamente uma pandemia. Suas manifestações variam entre assintomáticas ou Síndrome Respiratória Aguda Grave (SARS). O vírus possui rápida transmissão e já deixou milhões de infectados e mortos em todo o mundo (VELAVAN; MEYER, 2020).

Nesse contexto de pandemia, se faz necessário salientar o indispensável papel da nutrição como uma ferramenta vital no contexto entre alimentação, obesidade e risco de infecção por COVID-19, justificando um estado de alerta à saúde, já que as doenças adjacentes da obesidade são as mesmas do grupo de risco para o novo coronavírus.

A obesidade é uma epidemia que vem triplicando desde 1975, afetando mais de 650 milhões de adultos e 124 milhões de crianças e adolescentes em todo o mundo (OMS, 2020). Essa prevalência é preocupante, pois a obesidade é reconhecida como uma patologia comumente associada a outras doenças crônicas e podem estar relacionada com os agravos do quadro clínico do paciente levando ao surgimento dos sintomas mais severos do Sars-CoV-2, possuindo assim, um alto impacto na esfera da saúde pública dado a alta taxa de obesos e a rápida disseminação e infecção do vírus (OMS, 2000; PASCARELLA et al., 2020).

Ponderando surtos infecciosos antigos como a epidemia do H1N1 em 2009, no qual a obesidade ficou reconhecida como fator predisponente e agravante para a infecção e complicações da mesma (JAIN et al., 2009; ASHBAUG et al., 2013). A relação entre obesidade e as manifestações clínicas mais bruscas do novo coronavírus ainda parecem incertas, portanto, estudos 
tentam explanar os vínculos fisiopatológicos efetivo e agravantes entre os pacientes obesos e infectados pela COVID-19 (CAUSSY et al., 2020; SIMONNET et al., 2020; MICHALAKIS; ILIAS, 2020).

Diante desse cenário, este estudo tem como objetivo realizar um levantamento bibliográfico sobre a possível correlação entre a obesidade e o COVID-19, assim como identificar nutrientes que possam auxiliar na prática profissional do nutricionista, visando uma conduta nutricional adequada para os pacientes.

\section{Metodologia}

Trata-se de uma revisão da literatura do tipo narrativa. Para isso, utilizou-se artigos, guias e documentos oficiais de organizações da área de saúde e nutrição em língua inglesa e portuguesa recuperados a partir da busca nos seguintes bancos de dados: PubMed, ScienceDirect, SciELO (Scientific Eletronic Library Online). Foram utilizados descritores em ciências da saúde (Decs): "Nutrition"; "food"; "obesity" e "COVID-19". Estes foram utilizados de forma isolada e combinada entre si.

Foram incluídos no estudo documentos publicados durante o período de dezembro de 2019 a março de 2021 que continham informações úteis destacando nutrientes, bem como uma prática nutricional adequada para auxiliar pacientes obesos e infectados com COVID-19. Foram excluídas teses, dissertações, monografias, artigos que estavam fora da delimitação temporal estabelecida, artigos duplicados entre os bancos de dados, além de documentos que não se detinham a temática explorada nesse estudo. Para inclusão os trabalhos foram filtrados quanto a qualidade da pesquisa e do conteúdo do manuscrito a partir da leitura minunciosa do título e resumo de artigos e filtrados novamente após leitura completa.

\section{Resultados e discusões}

Nesse estudo foram analisados 85 artigos, no qual 57 foram selecionados por atender todos os critérios de inclusão e conter informações relevantes para a construção do trabalho. 


\subsection{Mecanismos Biológicos entre Obesidade e COVID-19}

Diante da atual pandemia de COVID-19 e seu alto risco de infecção é prudente considerar os possíveis mecanismos pelos quais o novo vírus pode impactar negativamente à saúde humana. A obesidade tem se mostrado um fator de risco em pacientes infectados com COVID-19 nos casos de gravidade e morte, pois o tecido adiposo pode se tornar um reservatório do SARS-CoV-2 causando disseminação a longo prazo, além disso o vírus demostra usar a mesma via de inflamação presente na obesidade prejudicando a resposta imunológica e afetando diretamente o prognóstico dos pacientes com COVID19 por ampliarem a resposta pró-inflamatória (CHIAPPETTA et al., 2020; LUZI; RADAELLI, 2020).

As condições associadas à obesidade parecem agravar o efeito da COVID-19, se origina de inclinações predisponentes como doenças crônicas, síndrome metabólica e desregulação imunológica (MAUVAIS-JARVIS, 2020; FANG et al., 2020; SATTAR, MCINNES, MCMURRAY., 2020) protagonizando um ambiente simbiótico propício para agravante como inflamação sistêmica crônica, comum em quadros de obesidade, ocasionada por secreções inflamatórias produzidas pelas citocinas do tecido adiposo, aumentando a quantidade de moléculas inflamatórias circulantes como linfócitos e macrófagos conduzindo uma cascata de eventos para tentar manter a homeostase celular e proteger o organismo (KIM et al., 2020).

A obesidade associada à infecção viral por SARS-CoV-2 desencadeia um quadro de inflamação crônica acentuada que diminui a capacidade do sistema imunitário de reconhecer e atuar de forma eficaz, como os linfócitos e células $B$ e T (responsáveis pela produção de anticorpos contra antígenos) causando um defeito na imunidade inata ( $\mathrm{CACl}$ et al., 2020), o que afeta as barreiras de defesa e aumenta a suscetibilidade a infecções graves, fator que expõe um possível meio pelo qual a obesidade é considerada um agravante nos casos de infecção pela COVID-19 (LUZI; RADAELLI, 2020). 


\subsection{Conduta nutricional para pacientes obesos infectados por COVID- 19 em estado grave}

Sabe-se que o comportamento e as emoções influenciam em nossas escolhas alimentares, influenciando no desejo de comer, principalmente, alimentos industrializados com alto teor de açúcares e gorduras, tornando baixa e até mesmo deficiente a ingestão de micronutrientes como vitaminas e minerais que são essenciais para as funções imunomoduladoras (SHAKOOR et al., 2020).

Junto a isso, de acordo com IDDIR et al., (2020), a deficiência de micronutrientes em indivíduos de algumas regiões está associada ao aumento nas taxas de obesidade, validando a alimentação não somente como uma terapia não medicamentosa, mas como uma possível associada à obesidade.

Os pacientes infectados pela COVID-19 em suas formas mais graves são vistos na Unidade de Terapia Intensiva (UTI) e são, frequentemente, idosos com sobrepeso ou obesos (THIBAULT et al., 2020). O manejo físico e nutricional adequado desses pacientes torna-se desafiante, pois o excesso de peso, juntamente com o estado de saúde fragilizado podem comprometer a autonomia do paciente quanto a tarefas simples como o autocuidado diário, refletindo seu quadro clínico debilitado.

O novo SARS-CoV-2 afeta não somente o sistema respiratório, como também tem demonstrado invadir o trato gastrointestinal (HUANG et al., 2019; XIÃO et al., 2020; CHEUNG et al., 2020).

O nutricionista é apontado como um importante profissional para o bom prognóstico do paciente, pois a partir da avaliação do estado nutricional e do grau de risco podem ser traçadas estratégias e condutas assertivas. A Sociedade Europeia de Nutrição Clínica e Metabolismo enfatiza que a nutrição como se caracteriza uma ferramenta imprescindível desde a prevenção ao tratamento e reabilitação do paciente (BARAZZONI et al., 2020).

A American Society for Parenteral and Enteral Nutrition, desde 1986 têm publicado diretrizes sobre o emprego da nutrição enteral como uma primeira e precoce forma de suporte nutricional que deve ser administrada 24 a 48 horas após a internação, por meio enteral ou parenteral (THIBAULT et al., 2020; MCKNIGHT et al., 2019) como forma de aplacar os desgastes rápidos que as patologias desempenham no metabolismo humano, visto que o excesso de 
peso é considerado um dos agravantes para a COVID-19 (STEFAN et al., 2020).

Ainda é um grande desafio elucidar as perguntas que permeiam as necessidades energéticas, metabólicas e nutritivas de cada paciente. Sendo assim, a análise clínica precisa ser criteriosa, a fim de evitar/tratar os eventos clínicos como hipercatabolismo proteico, possível desenvolvimento de sarcopenia como consequência da perda muscular esquelética, aumento de inflamação e estresse oxidativo, reprimindo maiores agravos que podem expressar a necessidade de um maior tempo na UTI (WANG et al., 2020).

Outra preocupação são sintomas referentes à admissão e fase aguda da COVID-19 que é a inflamação e a falta de apetite (HOEHL, 2020) que reduz a ingestão alimentar e aumenta o perigo da Síndrome de Realimentação (RFS) caracterizada por distúrbios metabólicos e eletrolíticos como desequilíbrio da glicose, deficiência de tiamina, hipocalemia e hipofosfatemia (MCKNIGHT et al., 2019).

O Ministério da Saúde, por meio da Fundação Oswaldo Cruz (2020) publicou um guia com dicas de higienização, ingredientes e informações para incentivar a população a manter práticas alimentares saudáveis durante 0 período de quarentena como uma forma de fortalecer o sistema imune. Essas informações estimulam o uso limitado de sal, açúcar e gorduras e incitam maior ingestão de macro e micronutrientes in natura.

Os nutrientes exercem um fator central no desempenho e competência imunológica, exemplo disso é a suscetibilidade ao desenvolvimento e aparecimento de doenças na deficiência de um ou mais micronutrientes e oligoelementos específicos da integridade funcional (GOMBART et al., 2020).

Diante das necessidades energéticas de um paciente infectado com 0 novo coronavírus e sua rápida replicação viral, a baixa quantidade de ingestão proteica resulta em uma diminuição da produção de anticorpos, reduzindo as defesas naturais, além de aumentar o risco de infecções mais graves. Portanto, a desnutrição proteica fragiliza o sistema imune e oportuniza 0 desenvolvimento de doenças gastrointestinais e respiratórias, o que explicaria ser o quadro clínico encontrado nos pacientes em estado grave com COVID-19 (BARAZZONI et al., 2020; VOLKERT, 2019). 


\subsection{Atuação e função protetora de micronutrientes no sistema imune}

As complicações geradas pela obesidade em concomitância com a COVID-19, geram alto comprometimento na saúde do indivíduo. A partir disso, a busca por soluções preventivas e terapêuticas que promovam qualidade de vida são de grande valia, já que os nutrientes exercem efeitos moduladores à saúde mediando maiores chances do indivíduo contra o vírus (AMAN; MASOOD, 2020).

Entre os nutrientes que apresentam função moduladora no sistema imune segundo a literatura estão as vitaminas $\mathrm{C}$ e $\mathrm{D}$ e oligoelementos como o zinco e o selênio.

\subsubsection{Vitamina C}

A vitamina $C$ é uma vitamina hidrossolúvel que desempenha uma gama de funções moduladoras sobre o sistema imunológico, famosa por sua atividade nos casos de infecções virais, na qual implica diretamente na redução das espécies reativas de oxigênio (EROS), maturação das células $T$, fagocitose e apoptose de neutrófilos extenuados. Adicionado a isso, pode contribuir na recuperação de pacientes com COVID-19, pois atenua a excessiva resposta anti-inflamatória (ZHITKOVICH, 2020; BAE, 2020).

Ressalta-se está presente em maior quantidade em células imunes como os leucócitos, do que no plasma sanguíneo. Em meio ao desequilíbrio oxidativo proporciona a ativação de moléculas pró-inflamatórias como o fator nuclear $\mathrm{kB}$ (NF-kB). Além de atuar como conivente coautora no controle de mediadores inflamatórios, podendo agravar ainda mais as condições inflamatórias em razão da retroalimentação, um ciclo constante que resulta em agravos (SHAKOOR et al., 2020).

A secreção de citocinas pró-inflamatórias como: IL-1 $1 \beta$, TNF- $\alpha$, IL-6 e IL-8 durante a resposta imunológica gerada a partir de estímulos virais e, consequentemente, pela COVID-19, podem apresentar um perfil inflamatório acentuado e depressor a saúde (SHAKOOR et al., 2020).

Estudos mostraram que os pacientes infectados com COVID-19 que foram tratados com altas doses de vitamina $C$ apresentaram uma recuperação 
mais rápida e sintomas reduzidos (KHAN et al., 2020; CHENG; SHANGHAI, 2020).

\subsubsection{Vitamina D}

A vitamina $D$ ou calciferol é lipossolúvel e desempenha papel importante sobre o organismo, no qual encontra-se em duas isoformas, ou seja, a vitamina D2 que deriva-se principalmente de fontes alimentares e a vitamina D3 obtida por meio da exposição solar a raios ultravioleta B (UVB) (CHAROENNGAM; HOLICK, 2020). Corroborando, pesquisadores destacam a atuação da vitamina D no sistema imunológico (BRIGUGLIO et al., 2020).

Com achados clínicos como o de D'avolio et al. (2020), tem se atribuído a vitamina D um considerável impacto sobre os pacientes infectados com SARSCoV-2, pois, foi demonstrado que pacientes com a Proteína C Reativa (PCR) positivos para o vírus, simultaneamente também eram abordados com níveis considerados inferiores de 25-hidroxivitamina D, principal metabólico circulante desta vitamina, sugerindo possível relação entre os achados.

Inferem-se em estudo que indivíduos de faixa etária avançada portam reduzidas concentrações de 25-hidroxivitamina $D$, portanto, são vulneráveis ao vírus, no qual contribui em menores sucessos das defesas e possibilita piores quadros da doença (CHANDRAN et al., 2020).

Em um estudo piloto, a administração de altas doses de calcifediol (25hidroxivitamina D 3) metabólito da vitamina $D$, em pacientes hospitalizados com a Covid-19, reduziu a necessidade de tratamento em UTI, enfatizando mais uma vez a vitamina $\mathrm{D}$ como mecanismo para mitigar os quadros referente ao vírus em questão (CASTILLO et al., 2020).

\subsubsection{Zinco}

O zinco possui propriedades imunomoduladoras e protetoras tornando-o de relevante interesse no contexto da pandemia do COVID-19. De acordo com Wen et al. (2020) uma das características marcantes da COVID-19 é o desequilíbrio imunológico, relacionado ao perfil inflamatório das vias aéreas, alvéolos e capacidade de trabalho mucociliar dos pulmões. 
Esssa condição pode ter sua causalidade em detrimento a baixa disponibilidade e desregulação no metabolismo do zinco, visto que níveis séricos adequados desse micronutriente é sugerido como estratégia para mitigar possíveis danos pulmonares (WESSEL et al., 2020).

Até o momento não existem evidências conclusivas na literatura sobre sua ação terapêutica no tratamento da covid, entretanto a cloroquina, sugerida para o tratamento da COVID-19 é um composto de ionóforo de zinco que aumenta as concentrações celulares e o fluxo intracelular de zinco que além de atividade anticâncer, pode contribuir presumivelmente para funções benéficas como um suporte na terapia de combate ao COVID-19 por seu alto poder antiviral (SKALNY et al., 2020).

Um estudo do National Institutes of Health (NIH) mostrou que a suplementação de zinco diminui a taxa de incidência da infecção do trato respiratório inferior em pacientes pediátricos, logo, a associação do zinco pode desempenhar um poder efetivo de combate ao vírus (RAHMAN et al., 2020; SKALNY et al., 2020). A replicação do SARS-CoV-2 pode ser prejudicada ou até inibida na presença de zinco intracelular com ionóforos de zinco, diminuindo desse modo a carga viral e auxiliando no tratamento (ZHANG; LIU, 2020).

Nesse contexto, a ingestão frequente de alimentos que contenham zinco torna-se indispensável para o bom funcionamento do organismo, pois ele participa de funções catalíticas e regulatórias, auxiliando no fortalecimento e manejo protetor do ambiente imunológico contra o COVID-19, além de possuir função mediadora da absorção de outros nutrientes como ferro. As recomendações diárias segundo a Recommended Dietary Allowance (RDA) são de 8-14 mg/dia (RERKSUPPAPHOL, 2019).

\subsubsection{Selênio}

O Selênio é um dos minerais que ganham destaque como co-fator, de alta capacidade, de vias antioxidante em humanos, tendo ação dependente da atuação de selenoproteinas específicas que exercem funções metabólicas, além de executar relevância na interação vírus-hospedeiro (WEN et al., 2020). 
Sua deficiência pode afetar não somente o hospedeiro, como também o patógeno presente durante alguma infecção viral aumentando sua virulência, comprometendo o sistema imune e contribuindo para sua alta taxa de replicação em virtude do estresse oxidativo originado (SCHOMBURG, 2020; GUILLIN et al., 2019).

Fogarty et al. (2020) descrevem que esse oligoelemento pode agir como integrante potencial no combate contra o COVID-19, em consonância a sua atuação direta ao sistema imune que intensifica respostas celulares e humorais aprimorando fenômenos de fagocitose mediada por células natural killer; proliferação dos linfócitos $T$ e síntese de imunoglobulinas na luta contra o vírus. Além de auxiliar na atuação das vitaminas $E$ e $D$, reduzindo também as suceptibilidades a formações de trombos sanguineos que podem ser fatais nos quadros de covid (SPYROPOULOS; WEITZ., 2020).

Estudos relacionam a influência nos níveis séricos do selênio com a sobrevivência de pacientes com COVID-19. Foi observado que os pacientes falecidos apresentavam deficiência de selênio em $64,7 \%$ e $70,6 \%$ dos infectados e mortos, enquanto os sobreviventes apresentavam taxas de deficiência de $39,3 \%$ e $32,6 \%$. Evidencia-se que quanto menor o status de selênio menor a taxa de recuperação e maior fator de risco para mortalidade pelo vírus (ZHANG et al., 2020; MOGHADDAM et al., 2020)

Pode-se denominar deficiência de selênio quando a ingestão diaria é $\leq a$ $11 \mu \mathrm{g}$ por dia com manifestações clínicas como fadiga muscular, deficit de concentração, doenças cardiovasculares, alterações no sistema imunológico, sistema endócrino, reprodutivo e psíquico. Segundo o requisito da RDA para a melhor atuação biológica e imunológica a ingestão diaria indicada é de 55 à $70 \mu \mathrm{g}$ por dia para homens e mulheres (ALEXANDER et al., 2020).

Entretanto, a Associação Brasileira de Nutrologia (ABRAN, 2020) recomenda que a quantidade de ingestão segura é de até 200 microgramas por dia e pode ser coadjuvante no tratamento de infecções, entretanto, niveis acima representam riscos de toxicidade. Para manter o suprimento adequado e suficiente de selênio manter uma alimentação equilibrada e variável contendo fontes alimentares ricas neste micronutriente. 


\section{Conclusão}

A partir dos dados obtidos, infere-se que a obesidade pode ser um agravante para COVID-19, isso ocorre devido ao perfil inflamatório comumente presente na obesidade que é ampliado pela COVID-19 levando a sérios problemas de saúde.

Não há evidências robustas que alimentos e/ou nutrientes isolados que possam combater ou prevenir contra o coronavírus. Entretanto, a ingestão adequada de vitaminas e minerais propiciam inúmeros benefícios à saúde, dentre eles funções antioxidantes, anti-inflamatórias, cofatores de enzimas para reações bioquímicas, equilíbrio oxidativo e fisiológico, atividade antiviral, maturação e proliferação de células do sistema imunitário. Assim, a inserção de uma conduta nutricional que vise um maior aporte de nutrientes essenciais como vitaminas e minerais pode ser uma estratégia interessante para fortalecimento do sistema imunológico.

\section{Referências}

ABRAN. Associação Brasileira de Nutrologia. A respeito de micronutrientes e prebióticos na infecção por COVID-19. 2020. Disponível em: https://abran.org.br/2020/05/01/posicionamento-da-associacao-brasileira-denutrologia-abran-a-respeito-de-micronutrientes-e-probioticos-na-infeccao-por-covid-19/ Acessado em 15 de Fev. 2021.

ALEXANDER, J. et al. Early nutritional interventions with zinc, selenium and vitamin D to increase antiviral resistance against progressive COVID-19. Nutrients, v. 12, n. 8, p. 2358, 2020.

AMAN, F.; MASOOD, S. How Nutrition can help to fight against COVID-19 Pandemic. Pakistan Journal of Medical Sciences, v. 36, n. COVID19-S4, 2020.

ASHBAUGH, A. R. et al. The decision to vaccinate or not during the H1N1 pandemic: selecting the lesser of two evils?. PLoS One, v. 8, n. 3, p. e58852, 2013.

$\mathrm{BAE}, \mathrm{M} ; \mathrm{KIM}, \mathrm{H}$. Mini-review on the roles of vitamin $\mathrm{C}$, vitamin $\mathrm{D}$ and selenium in the immune system against COVID-19. Molecules, vol. 25, n. 22, p. 5346, 2020.

BARAZZONI, R. et al. ESPEN expert statements and practical guidance for nutritional management of individuals with SARS-CoV-2 infection. Journal of Clinical Nutrition, v. 40, n. 2 , p. 339-660, 2020.

BRIGUGLIO, M. et al. The malnutritional status of the host as a virulence factor for new coronavirus SARS-CoV-2. Frontiers in medicine, v. 7, p. 146, 2020.

CACI, Grazia et al. COVID-19 and obesity: dangerous liaisons. Journal of clinical medicine, v. 9, n. 8, p. 2511, 2020. 
CASTILLO, M. et al. Effect of calcifediol treatment and best available therapy versus best available therapy on intensive care unit admission and mortality among patients hospitalized for COVID-19: A pilot randomized clinical study. The Journal of steroid biochemistry and molecular biology, v. 203, p. 105751, 2020.

CAUSSY, C. et al. Obesity is associated with severe forms of COVID-19. Obesity, v. 28, n. 7, p. 1175-1175, 2020.

CHANDRAN, M. et al. Vitamin D in COVID-19: Dousing the fire or averting the storm?A perspective from the Asia-Pacific. Osteoporosis and Sarcopenia, 2020. V. 6, n. 3, p. $97-105,2020$.

CHAROENNGAM, N; HOLICK, M. F. Immunologic effects of vitamin D on human health and disease. Nutrients, v. 12, n. 7, p. 2097, 2020.

CHENG, R. Z. Can an early and high intravenous dose of vitamin $\mathrm{C}$ prevent and treat coronavirus disease 2019 (COVID-19)? Medicine in Drug Discovery, v. 5, p. 100028, 2020.

CHEUNG, K. S. et al. Gastrointestinal manifestations of SARS-CoV-2 infection and virus load in fecal samples from a Hong Kong cohort: systematic review and metaanalysis. Gastroenterology, v. 159, n. 1, p. 81-95, 2020.

CHIAPPETTA, S. et al. COVID-19 and the role of chronic inflammation in patients with obesity. International Journal of Obesity, v. 44, p. 1790 - 1792, 2020.

DRUYAN, M. E. et al. Clinical guidelines for the use of parenteral and enteral nutrition in adult and pediatric patients: applying the GRADE system to development of ASPEN clinical guidelines. JPEN J Parenter Enteral Nutrition v. 36, n. 1, p. 77-80, 2012.

FOGARTY, H. et al. COVID19 coagulopathy in Caucasian patients. British Journal of Hematology, V. 189, n. 6, p. 1044 - 1049. 2020.

GOMBART, A. F.; PIERRE, A.; M, Silvia. A review of micronutrients and the immune system-working in harmony to reduce the risk of infection. Nutrients, v. 12, n. 1, p. 236, 2020.

Guia de orientações em relação à alimentação e exercício físico diante da Covid-19.

Núcleo de Alimentação, Saúde e Ambiente - Nasa/CST/Cogepe/Fiocruz. $4^{\circ}$ edição. Rio de Janeiro. 2020.

GUILLIN, O. M. et al. Selenium, selenoproteins and viral infection. Nutrients, v. 11, n. 9, p. 2101, 2019.

HOEHL, S. et al. Evidence of SARS-CoV-2 infection in travelers returning from Wuhan, China. New England Journal of Medicine, vol. 382, n. 13, p. 1278-1280, 2020.

HUANG, C. et al. Clinical features of 2019 new coronavirus-infected patients in Wuhan, China. The lancet, v. 395, n. 10223, p. 497-506, 2020.

IDDIR, M. et al. Strengthening the immune system and reducing inflammation and oxidative stress through diet and nutrition: considerations during the COVID-19 crisis.

Nutrients, v. 12, n. 6, p. 1562, 2020. 
JAIN, S. et al. Patients hospitalized with H1N1 influenza 2009 in the United States, April-June 2009. New England Journal of Medicine, v. 361, n. 20, p. 1935-1944, 2009.

JEAN, G.; SOUBERBIELLE, J. C.; CHAZOT, C. Vitamin D in chronic kidney disease and dialysis patients. Nutrients, v. 9, n. 4, p. 328, 2017.

KHAN, $\mathrm{H}$. et al. Unusual early recovery of a critical patient with COVID-19 after intravenous vitamin $\mathrm{C}$ administration. The American Journal of Case reports, v. 21, p. 925521-1, 2020.

$\mathrm{KIM}$, J. et al. What we know and what we need to know about adenovirus 36 -induced obesity. International Journal of Obesity, p. 1-13, 2020.

LESSA, I. Doenças crônicas não-transmissíveis no Brasil: um desafio para a complexa tarefa da vigilância. Ciência \& Saúde Coletiva, v. 9, p. 931-943, 2004.

LIU, P. T. et al. Toll-like receptor triggering of a vitamin D-mediated human antimicrobial response. Science, v. 311, n. 5768, p. 1770-1773, 2006.

LUZI, L; RADAELLI, M. G. Influenza and obesity: its odd relationship and the lessons for COVID-19 pandemic. Acta Diabetologica, p. 1-6, 2020.

MAUVAIS-JARVIS, F. Aging, male sex, obesity, and metabolic inflammation create the perfect storm for COVID-19. Diabetes, v. 69, n. 9, p. 1857-1863, 2020.

MCKNIGHT, C. L. et al. Refeeding syndrome in the critically ill: a literature review and clinician's guide. Current gastroenterology reports, v. 21, n. 11, p. 58, 2019.

MICHALAKIS, K; ILIAS, I. SARS-CoV-2 infection and obesity: Common inflammatory and metabolic aspects. Diabetes \& Metabolic Syndrome: Clinical Research \& Reviews, 2020.

MOGHADDAM, A. et al. Selenium deficiency is associated with the risk of mortality from COVID-19. Nutrients, v. 12, n. 7, p. 2098, 2020.

MUELLER, C. et al. ASPEN clinical guidelines: nutritional screening, evaluation and intervention in adults. Journal of Parenteral and Enteral Nutrition, vol. 35, n. 1, p. 16-24, 2011.

MUSCOGIURI, G. et al. Nutritional recommendations for COVID-19 quarantine. European Journal of Clinical Nutrition, n. 74, p. 1-2, 2020.

MUSCOGIURI, Giovanna et al. Comentary: obesity: the "Achilles heel" for COVID-19?. Metabolism-Clinical and Experimental, v. 108, 2020.

PASCARELLA, Giuseppe et al. COVID-19 diagnosis and management: a comprehensive review. Journal of internal medicine, v. 288, n. 2, p. 192-206, 2020.

RAHMAN, M. A. et al. Efficacy of the green synthesized nickel-oxide nanoparticles against pulse beetle, Callosobruchus maculatus (F.) in black gram (Vigna mungo L.). International Journal of Pest Management, p. 1-9, 2020. 
RERKSUPPAPHOL, S.; RERKSUPPAPHOL, L. A randomized controlled trial of zinc supplementation in the treatment of acute respiratory tract infection in Thai children.

Pediatric reports, v. 11, n. 2, 2019.

SALTIEL, Alan R. et al. Inflammatory mechanisms linking obesity and metabolic disease. The Journal of clinical investigation, v. 127, n. 1, p. 1-4, 2017.

SATTAR, Naveed; MCINNES, lain B.; MCMURRAY, John JV. Obesity is a risk factor for severe COVID-19 infection: multiple potential mechanisms. Circulation, v. 142, n. 1, p. 4-6, 2020.

SCHOMBURG, L. The other view: the trace element selenium as a micronutrient in thyroid disease, diabetes and beyond. Hormones, p. 1-10, 2020.

SHAKOOR, $\mathrm{H}$. et al. Immune-boosting role of vitamins $\mathrm{D}, \mathrm{C}$, E, zinc, selenium and omega-3 fatty acids: could they help against COVID-19?. Maturitas, 2020.

SIMONNET, A. et al. High prevalence of obesity in severe acute respiratory syndrome coronavirus-2 (SARS-CoV-2) requiring invasive mechanical ventilation. Obesity, 2020.

SKALNY, A. V. et al. Zinc and respiratory tract infections: Perspectives for COVID-19. International Journal of Molecular Medicine, v. 46, n. 1, p. 17-26, 2020.

SPYROPOULOS, A. C.; WEITZ, J. I. Hospitalized COVID-19 patients and venous thromboembolism: a perfect storm. Circutation, v. 142, n. 2, p. 129-132, 2020.

STEFAN, N. et al. Obesity and impaired metabolic health in patients with COVID-19. Nature Reviews Endocrinology, p. 1-2, 2020.

THIBAULT, R. et al. Nutrition of the COVID-19 patient in the intensive care unit (ICU): a practical guidance. Critical Care, v. 24, n. 1, p. 1-8, 2020.

VOLKERT, D. et al. ESPEN guideline on clinical nutrition and hydration in geriatrics. Clinical Nutrition, v. 38, n. 1, p. 10-47, 2019.

WANG, D. et al. Clinical characteristics of 138 hospitalized patients with 2019 novel coronavirus-infected pneumonia in Wuhan, China. Jama, v. 323, n. 11, p. 1061-1069, 2020.

WEN, W. et al. Immune cell profiling of COVID-19 patients in the recovery stage by single-cell sequencing. Cell discovery, v. 6, n. 1, p. 1-18, 2020.

WESSELS, I. et al. Zinc supplementation ameliorates lung injury by reducing neutrophil recruitment and activity. Thorax, v. 75, n. 3, p. 253-261, 2020.

WORLD HEALTH ORGANIZATION (WHO). Obesity and overweight. 2020. https://www.who.int/news-room/fact-sheets/detail/obesity-and-overweight.

WORLD HEALTH ORGANIZATION. Obesity: preventing and managing the global epidemic. Report of a WHO consultation on obesity. Geneva, Switzerland: WHO, 2000.

$\mathrm{XIAO}$, F. et al. Evidence for gastrointestinal infection of SARS-CoV-2.

Gastroenterology, v. 158, n. 6, p. 1831-1833, 2020. 
ZHANG, L; LIU, Y. Potential interventions for novel coronavirus in China: A systematic review. Journal of medical virology, v. 92, n. 5, p. 479-490, 2020.

ZHITKOVICH, A. Nuclear and Cytoplasmic Functions of Vitamin C. Chemical research in toxicology, v. 33, n. 10, p. 2515-2526, 2020. 